\title{
Secondary Wall Formation in Fibers of Arabidopsis thaliana
}

\author{
E.A. Richardson, G.-K. Zhou, R. Zhong, C.J. Nairn, and Z.-H. Ye \\ University of Georgia, Department of Plant Biology, Athens, GA 30602
}

Secondary walls are the major constituents of tracheary elements and fibers in wood. They typically are composed of three distinct layers of cellulose microfibrils, namely S1, S2 and S3 layers. The major components of secondary walls from fibers are cellulose, lignin, and hemicelluloses including xylan and glucomannan [1]. Although the genes participating in the biosynthesis of cellulose and lignin have been studied intensively, genes involved in xylan synthesis are poorly understood. Because xylan is the second most abundant polysaccharide after cellulose in wood, understanding how xylan is synthesized is not only important for basic plant biology but also is essential for potential genetic improvements of the quality and quantity of wood.

We are interested in uncovering genes involved in the biosynthetic pathway of xylan in fibers [2] and recently have found that two novel glycosyltransferases, GT1 and GT2, are essential for normal secondary wall formation in $A$. thaliana fibers. A. thaliana inflorescence stems develop interfascicular fibers for mechanical support of shoots. Mutations of the GT1 and GT2 genes cause a dramatic reduction in stem strength. Cross sections of the mutant stems showed that interfascicular fibers have much thinner walls compared with the wild type (Figs. 1, 2). In addition, xylem vessels are severely collapsed probably due to the reduced wall strength that renders them susceptible to the negative pressure generated during transpiration. Transmission electron microscopy further revealed that secondary wall thickness in both fibers and vessels of the mutants was reduced drastically when compared with the wild type (Figs. 3, 4). These results clearly demonstrate that both GT1 and GT2 are involved in secondary wall synthesis in fibers and vessels.

The drastic reduction in secondary wall thickness caused by the GT1 and GT2 mutations suggests that they are involved in the biosynthesis of either cellulose or xylan. The cellulose-synthesizing machinery is located in the plasma membrane, and xylan is synthesized in the Golgi apparatus [1]. Since we found that yellow fluorescent protein (YFP) tagged GT1 and GT2 glycosyltransferases are localized in the Golgi apparatus (Figs. 5-8) where xylan is synthesized, we reason that GT1 and GT2 are involved in xylan synthesis. Our study of the GT1 and GT2 glycosyltransferases hopefully will lead to a better understanding of how plants make secondary walls in wood, the most abundant biomass produced by plants.

[1] N.C. Carpita and M.C. McCann, The Cell Wall. In Biochemistry and Molecular Biology of Plants, B.B. Buchanan, W. Gruissem, R.L. Jones, eds (Rockville, Maryland: American Society of Plant Physiologists), (2000) 52-108.

[2] R. Zhong et al., Plant Cell 17(2005) 3390.

[3] This work is supported by a grant from the U.S. Department of Energy-Bioscience Division. 


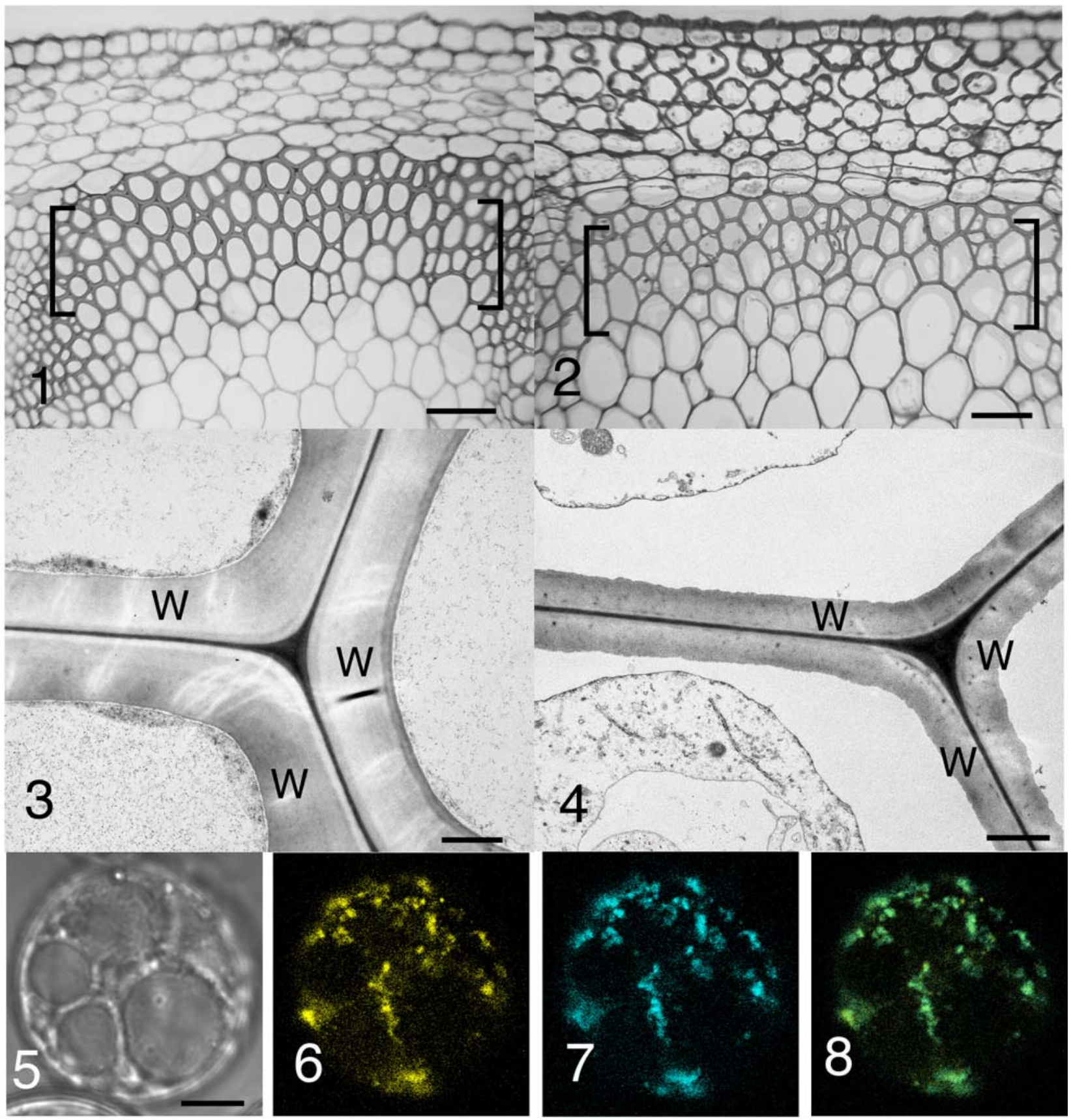

Figs. $1 \& 2$. Cross sections of interfascicular regions (brackets) of $A$. thaliana stems showing fiber cells with thin walls in the GT1 mutant (Fig. 2) compared with the wild type (Fig. 1). Bar $=30 \mu \mathrm{m}$.

Figs. $3 \& 4$. Transmission electron micrographs of fiber cell walls (W) showing a drastic reduction in their thickness in the GT1 mutant (Fig. 4) compared with the wild type (Fig. 3). Bar $=1 \mu \mathrm{m}$.

Figs. 5 to 8. DIC image (Fig. 5) of a carrot cell that expresses the GT1-yellow fluorescent protein (Fig. 6) and the Golgi-localized FRA8-cyan fluorescent protein (Fig. 7). A merged image (Fig. 8) of Figs. $6 \& 7$ shows that the localization pattern of GT1-yellow fluorescent protein is identical to that of FRA8-cyan fluorescent protein. Bar $=12 \mu \mathrm{m}$. 